\title{
Die feline Osteoarthrose
}

\author{
Alexander Acker, Sabine Tacke
}

\section{Teil 1: Grundlagen und Literatur}

Heutzutage weiß man, dass die Osteoarthrose (OA) eine häufige Erkrankung v. a. bei älteren Katzen ist. Dennoch stellt die Diagnosestellung immer noch ein großes Problem dar, da nicht die Lahmheit das Hauptsymptom ist, sondern Verhaltensänderungen. Daher nimmt ein ausführliches Besitzergespräch eine Schüsselrolle im Rahmen der Arthrosediagnostik bei der Katze ein.

\section{Gelenkaufbau und Physiologie}

Die an einem Gelenk beteiligten Strukturen sind die Gelenkkapsel, die aus dem Stratum fibrosum und dem Stratum synoviale besteht, der Gelenkknorpel, die Synovia sowie Knochen und Bänder.

\section{Gelenkkapsel}

Das aus straffem kollagenem Bindegewebe aufgebaute Stratum fibrosum dient zusammen mit den Bändern der mechanischen Stabilisation des Gelenks und geht im Ansatzbereich ins Periost über. Es enthält zahlreiche Blut- und Lymphgefäße wie auch Nerven. Dem Stratum fibrosum liegt innen das Stratum synoviale auf.

Das Stratum synoviale besteht aus mehreren Lagen Synovialozyten, die jedoch kein echtes Epithel bilden, da die typischen Zellkontakte und eine Basalmembran fehlen. Man unterscheidet:

Synovialozyten vom Typ A:

- für die Phagozytose von Bedeutung
Synovialozyten vom Typ B:

- sezernieren wichtige Synoviabestandteile wie u.a. Hyaluronsäure, Lumbricin o. Ä.

Zur Oberflächenvergrößerung bilden die Synovialozyten Synovialzotten aus, die ebenfalls Blutgefäße und Nervenendigungen enthalten.

\section{Synovia}

Die Synovia stellt ein Ultrafiltrat des Blutplasmas dar. Filtration bzw. Resorption hängen vom kolloidosmotischen und hydrostatischen Druck im Gelenk ab, sodass z.B. je nach Gelenkfüllung, Gelenkbeugung oder Belastung des Gelenks Filtration oder Rückresorption gefördert werden.

In der Synovia sind auch zahlreiche Zellen (Synovialo-, Mono- und Lymphozyten und z.T. auch Granulozyten) enthalten.
Hauptfunktion der Synovia ist die Ernährung des Gelenkknorpels und Reduktion der Reibung im Gelenk, hierzu ist eine hohe Konzentration an Hyaluronsäure wichtig.

\section{Gelenkknorpel}

Der Gelenkknorpel besitzt einzigartige biologische und mechanische Eigenschaften, diese beruhen auf seinem Aufbau und dem Zusammenwirken von Chondrozyten und der Extrazellulärmatrix.

Hauptbestandteil des Gelenkknorpels ist Wasser, dessen Gehalt beim adulten Tier ca. $70 \%$ beträgt, beim Neonaten kann der Wassergehalt sogar bis zu 90\% betragen. Die Trockensubstanz besteht aus Kollagen (v.a. Typ 2), Proteoglykan (35\%), Glykoproteinen (10\%), Mineralien (3\%) und Chondrozyten, deren Gehalt zwischen 1 und $15 \%$ schwanken kann.

\section{Definition Osteoarthrose}

Der Begriff Osteoarthrose bezeichnet einen Krankheitsprozess, der durch Degeneration des Gelenkknorpels, Knochenzubildungen an den Gelenkkapselrändern sowie Veränderungen der Synovialmembran charakterisiert ist.

Die Arthrose ist im deutschsprachigen Raum als eine primär nicht-entzündliche Erkrankung definiert. Sobald jedoch klinische Symptome vorhanden sind, ist die OA stets mit einer sterilen Entzündung verbunden, welches dem angloamerikanischen Begriff „osteoarthritis“ eine Berechtigung gibt.

Pathogenetisch wird die Osteoarthrose in eine primäre und sekundäre Form eingeteilt. Bei der primären, auch idiopathische Form genannt, ist keine direkte Ursache festzustellen. Der sekundären Form liegt stets eine Ursache wie z. B. eine Gelenkfraktur oder auch eine Hüftgelenksdysplasie zugrunde. 
Die Chondrozyten dienen der Sekretion, Regulation und Organisation der Extrazellulärmatrix. Die Ernährung der Knorpelzellen erfolgt über die Synovia, da der Knorpel keine Blutgefäße enthält.

Äste peripherer Nerven innervieren die Gelenkkapsel und dienen zum einen der Propriozeption und zum anderen der Nozizeption. Unter Nozizeption versteht man die Aufnahme (Transduktion), Weiterleitung (Transmission), zentrale Verarbeitung (Modulation) und bewusste Wahrnehmung (Perzeption) von noxischen Reizen. Diese Reize werden von den Nozizeptoren wahrgenommen und zum Dorsalhorn des Rückenmarks weitergeleitet. Vom Dorsalhorn erfolgt die Reizweiterleitung über den Tractus spinothalamicus zum Thalamus und Kortex. Im Kortex kommt es dann zur Schmerzwahrnehmung.

\section{Pathophysiologie}

Im Zentrum der Pathogenese der OA steht eine sterile Entzündung des Stratum synoviale der Gelenkkapsel, eine sog. Synovialitis.

Im Rahmen der Synovialitis kommt es zu einer vermehrten Freisetzung von Entzündungsmediatoren (z.B. Prostaglandine, Leukotrienen, Substanz P, Zytokine), von lysosomalen Enzymen, einer vermehrten Bildung von freien Radikalen durch Makrophagen und z.T. auch neutrophilen Granulozyten sowie zu einer vermehrten Aktivierung von Matrix-Metalloproteasen (MMPs).
Der Stoffwechsel des Knorpels wird streng kontrolliert. Es werden anabole Faktoren (z.B. IGF, TGF $\beta$ ), die für die Aufrechterhaltung, und katabole Faktoren (z.B. Il-1, TNF $\alpha$, Proteasen), die für den Abbau des Knorpels verantwortlich sind, unterschieden. Anabole und katabole Faktoren stehen unter physiologischen Bedingungen im Gleichgewicht zueinander, zudem wird die Aktivität der katabolen Faktoren durch sog. Regulatoren (z.B. Il-4 oder -6, ProteaseInhibitoren) sehr streng reguliert.

Durch eine Synovialitis, ein Trauma oder eine Instabilität kommt es zur Verschiebung dieses Verhältnisses zugunsten der katabolen Faktoren ( Abb. 1).

MMPs sind im gesunden Knorpel für den kontrollierten Abbau der Knorpelmatrix im Rahmen von physiologischen Umbauprozessen wichtig.

Zu der Gruppe der MMPs gehören Kollagenasen (MMP-1, MMP-8, MMP-13), Gelatinasen (MMP-2, MMP-9), Stromelysine (MMP-3, MMP-7, MMP-10, MMP-11), aber auch andere Proteasen wie z.B. Aggrekanasen, Elastasen oder Kathepsine, die bei der OA vermehrt freigesetzt werden. Als Substrat dienen ihnen u.a. Proteoglykane, Kollagen, Aggrekan, aber auch Fibrin und Plasminogen.

Diese Veränderungen führen zur Zunahme von Entzündungszellen in der Kapsel und der Synovia, zur Gefäßproliferation, Synovialzottenhyperplasie, Gelenkfüllung, Sensibilisierung der Nozizeptoren und Veränderungen im Knorpelmetabolismus.
Dies wirkt sich v.a. auf die Hauptbestandteile des Gelenkknorpels aus, wie dem Kollagen (v.a. Typ II), den Proteoglykanen, den Chondrozyten und dem Wassergehalt. Es kommt zu einem verminderten Umsatz von Kollagen, was sich negativ auf die Knorpelstabilität auswirkt, der Gehalt an Proteoglykanen nimmt ab, was wiederum die biomechanischen Eigenschaften des Knorpels beeinträchtigt.

Des Weiteren kommt es zur Degeneration von Chondrozyten, die für die Matrixerhaltung verantwortlich sind, und es kommt zu einer vermehrten Einlagerung von Wasser. Diese Veränderungen führen zu einer Chondromalazie mit einem weichen und weniger belastbaren Knorpel. Es kommt zu einer Destruktion des Knorpels, und die dabei entstehenden Knorpeldebris verstärken wiederum den Entzündungsprozess, was wiederum zu einer Zunahme der Knorpeldegeneration bis hin zum vollständigen Verlust des Gelenkknorpels führt.

Die Entzündungsprozesse sind an der Schmerzentstehung bei der OA von entscheidender Bedeutung, obwohl der Ursprung des Schmerzes noch nicht in allen Punkten genau bekannt ist. Der Knorpel selber ist nicht innerviert, aber Schmerzen können durch die Freisetzung von Mediatoren, wie z.B. Prostaglandine, Leukotriene, Substanz P und andere Zytokine, verursacht werden.

Diese Mediatoren bewirken eine Absenkung der Erregungsschwelle von Nozizeptoren in der Gelenkkapsel. Mit Fortschreiten des

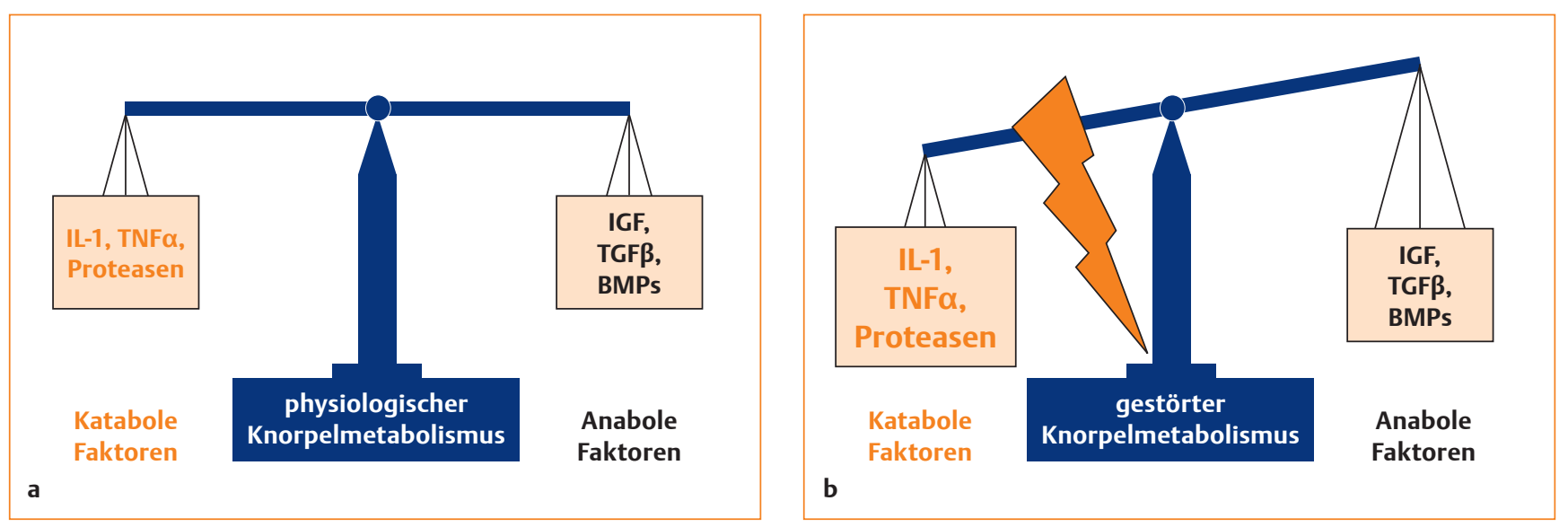

Abb. 1 a Schema: Knorpelmetabolismus. Im gesunden Gelenk stehen anabole und katabole Faktoren im ausgeglichenen Verhältnis zueinander. b Schema: gestörter Knorpelmetabolismus. Durch einen Insult (Trauma, Synovialitis etc.) entsteht eine Verschiebung zugunsten der katabolen Faktoren. 
Krankheitsbildes reichen oft immer geringere Reize aus, um die Nozizeptoren zu depolarisieren und Schmerzen zu verursachen (periphere Sensibilisierung). Die Sensibilisierung kann zu Hyperalgesie (verstärkte Reaktion auf schmerzhaften Reiz), Allodynie (schmerzhafte Empfindung eines nicht schmerzhaften Reizes) und der Entstehung eines Schmerzgedächtnisses führen.

Des Weiteren sind eine subchondrale Knochenbelastung, Osteophyten, die das Periost reizen, und übermäßige Belastung von Bändern und Gelenkkapsel an der Schmerzentstehung im Rahmen der OA beteiligt.

Die Pathogenese der Arthrose ist komplex und als eine Art Teufelskreis zu sehen ( Abb. 2).

\section{Epidemiologie}

Jüngere Studien zeigen, dass Katzen weitaus häufiger als früher angenommen von OA betroffen sind. Je nach Studie finden sich röntgenologische Hinweise bei bis zu $90 \%$ der Tiere.

Bei einer Untersuchung von Godfrey (2005) liegt die röntgenologische Arthroseprävalenz bei Katzen mit einem mittleren Alter von 9,5 Jahren allein im Bereich des Gliedmaßenskelettes bei $22 \%$.

Im Rahmen einer Studie von Hardie und Mitarbeitern (2002) fanden sich bei $90 \%$ der Katzen über 12 Jahren röntgenologische Hinweise auf das Vorliegen einer Osteoarthrose:
- bei $26 \%$ ausschließlich im Bereich der Wirbelsäule
- bei $10 \%$ ausschließlich am Gliedma-
ßenskelett
- bei $54 \%$ sowohl an der Wirbelsäule
als auch am Gliedmaßenskelett

$\mathrm{Zu}$ erwähnen ist jedoch, dass es sich bei diesen Zahlen um die radiologische und nicht die klinische Häufigkeit der felinen OA handelt und dass Röntgenbefunde nicht mit den Befunden der klinischen Untersuchung übereinstimmen müssen.

\section{Im Unterschied zur Situation beim Hund scheint die Mehrzahl der Osteoarthrosefälle bei der Katze primären Ursprungs zu sein.}

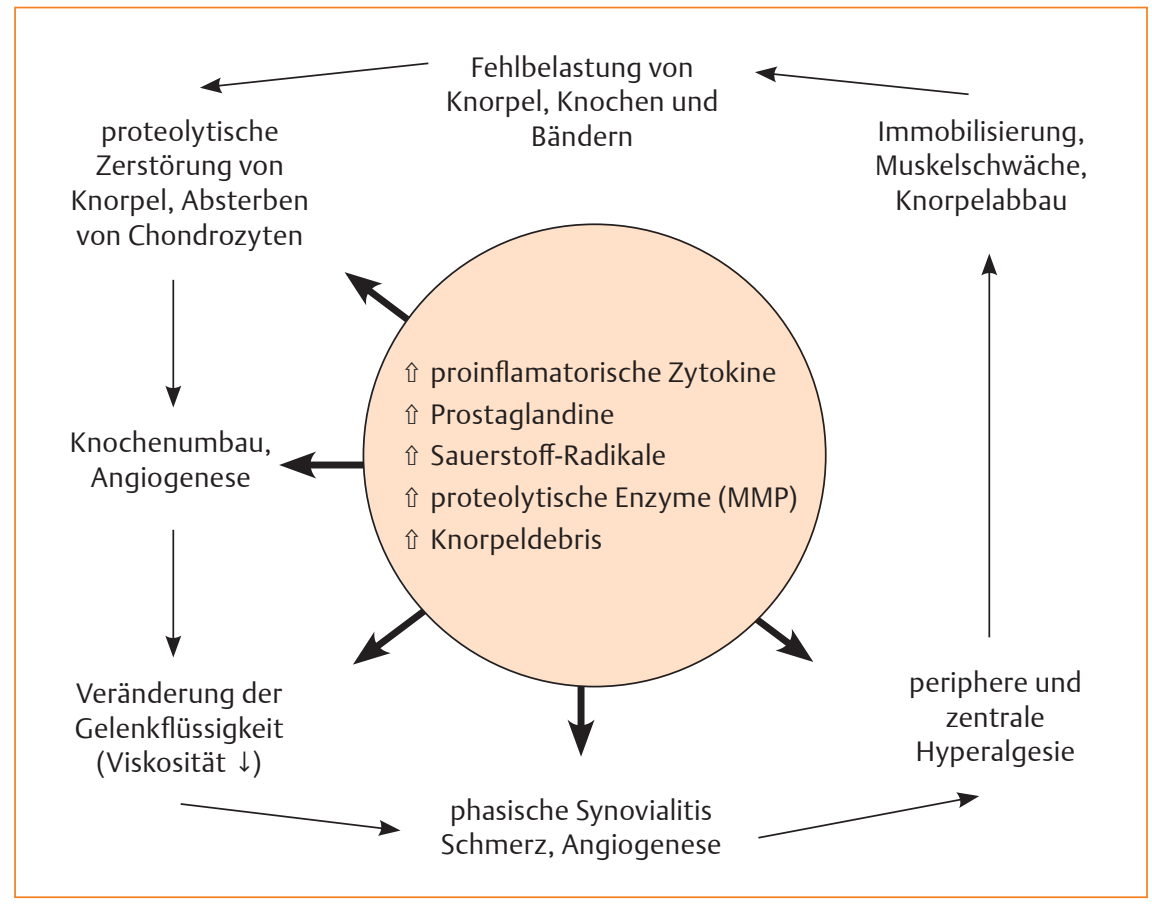

Abb. 2 Teufelskreis des Gelenkschmerzes.

Auffallend ist jedoch, dass arthrotische Veränderungen häufig bilateral auftreten, was charakteristisch für kongenitale Malformationen (z.B. Gelenksdysplasie), systemische Veränderungen (z.B. Endokrinopathien, Stoffwechselerkrankungen) oder Überbelastung ist.

Daher ist es fraglich, ob idiopathische Arthrosen tatsächlich in einem so hohen Maße vorkommen oder ob es bislang noch unbekannte Faktoren wie z.B. chronisch geringgradige Traumata oder dezente Malartikulation gibt, die Ursache für die Arthroseentstehung sein können.

\section{Literatur}

Allan GS. Radiographic features of feline joint disease. Vet Clin North Am Small Anim Pract 2000; 30(2): 281-302

Bennet D, Nash AS. Feline immune based polyarthritis: a study of thirty-one cases. J Small Anim Pract 1988; 29: 501-523

Caney S. Arthritis/Arthrose bei der Katze. Veterinary Focus 2007; 17(3): 11-17

Clarke SP, Bennett D. Feline osteoarthritis: a prospective study of 28 cases. J Small Anim Pract 2006; 47 : 439-445

Clarke SP, Mellor D, Clements N et al. Prevalence of radiographic signs of degenerative joint disease in a hospital population of cats. Vet Rec 2005; 157: 793-799

Erhardt W, Henke J, Haberstroh J. Anästhesie \& Analgesie beim Klein- und Heimtier sowie bei Vögeln, Reptilien, Amphbien und Fischen. Stuttgart: Schattauer Verlag: 2004

Godfrey DR. Osteoarthritis in cats: a retrospective radiological study. J Small Anim Pract 2005; 46: 425-429

Gunew MN, Menrath VH, Marshall RD. Long-term safety, efficacy and palatability of oral meloxicam at $0,01-$ $0,03 \mathrm{mg} / \mathrm{kg}$ for treatment of osteoarthritic pain in cats. J Feline Med Surg 2008; 10: 235-241
Handwerker HO. Einführung in die Pathophysiologie des Schmerzes. Heidelberg, Berlin: Springer Verlag; 1999 Hardie EM. Management of Osteoarthritis in cats. Vet Clin North Am Small Anim Pract 1997; 27(4): 945-953

Hardie EM, Roe SC, Martin FR. Radiographic evidence of degenerative joint disease in geriatric cats: 100 cases (1994-1997). J Am Vet Med Assoc 2002; 220(5): 628-632

Hoots EA, Petersen SW. Tibial plateau leveling osteotomy and cranial closing wedge ostectomy in a cat with cranial cruciate ligament rupture. J Am Anim Hosp Assoc 2005; 41(6): 395-399

Horzinek MC, Schmidt V, Lutz H. Krankheiten der Katze. Stuttgart: Enke Verlag; 2003

Kasper M, Zohmann A. Ganzheitliche Schmerztherapie für Hund und Katze. Stuttgart: Sonntag Verlag; 2007

Keller GG, Reed AL, Lattimer JC et al. Hip dysplasia: a feline population study. Vet Radiol Ultrasound 1999; 40(4): 460-464

Köppel E, Ebner J. Die Hüftgelenkdysplasie der Katze. Kleintierpraxis 1990; 35: 281-298

Langenbach A, Green P, Giger P et al. Relationship between degenerative joint disease and hip joint laxity by use of distraction index and Norberg angle measurement in a group of cats. J Am Vet Med Assoc 1998; 213(10): 1439-1443

Lascelles BDX, Court MH, Hardie EM, Robertson SA. Nonsteroidal anti-inflammatory drugs in cats: a review. Vet Anaesth Analg 2007; 34: 228-250

Lascelles BDX, Hansen BD, Roe $S$ et al. Evaluation of clientspecific outcome measures and activity monitoring to measure pain relief in cats with osteoarthritis. J Vet Intern Med 2008; 21(3): 410-416

Lascelles BDX, Hansen BD, Thomson A et al. Evaluation of a digitally integrated accelerometer-based activity monitor for the measurement of activity in cats. Vet Anaesth Analg 2008; 35: 173-173

Lascelles BDX. Osteoarthritis in cats - Myth or major problem. Proceedings of the Southern European Veterinary Conference \& Congreso Nacional AVEPABarcelona, Spain; 2009

Lascelles BDX, Henry JB, Brown J et al. Cross-Sectional Study of the Prevalence of Radiographic Degenerative Joint Disease in Domesticated Cats. Vet Surg 2010; 39: 535-544

Mathews KA. Nonsteroidal anti-inflammatory analgesics. Indications and contraindications for pain management in dogs and cats. Vet Clin North Am Small Anim Pract 2000; 30(2): 783-804 
Montavon PM, Voss K, Langley-Hobbs SJ. Feline Orthopedic Surgery and Musculoskeletal Disease. München: Elsevier Verlag; 2009

Nickel R, Schummer A, Seiferle E. Lehrbuch der Anatomie der Haustiere. Bd. 1: Bewegungsapparat. 7. Aufl. Berlin: Parey Verlag; 2001: 215-217

Patsikas MN, Papazoglou LG, Komminou A et al. Hip dysplasia in the cat: a report of three cases. J Small Anim Pract 1998; 39: 290-294

Pschyrembel. Klinisches Wörterbuch. 259. Aufl. Berlin: de Gruyter Verlag; 2002

Rivière S. Physiotherapie bei Katzen und Hunden mit Arthrose. Veterinary Focus 2007; 17(3): 32-36
Sanderson RO, Beata C, Flipo RM et al. Systematic review of the management of canine osteoarthritis. Vet Rec 2009; 164(14): 418-424

Scarlett JM, Donoghue S. Association between body condition and disease in cats. J Am Vet Med Assoc 1998; 212: 1725-1731

Slatter D, ed. Textbook of Small Animal Surgery. 3rd ed. Philadelphia: W.B. Saunders; 2003

Staiger BA, Beale BS. Use of arthroscopy for debridement of the elbow joint in cats. I Am Vet Med Assoc 2005: 226: 401-403

Siegenthaler W, Blum HE. Klinische Pathophysiologie. Stuttgart: Thieme Verlag; 2006
Tacke S, Schimke E, Kramer M et al. Resektionsarthroplastik des Hüftgelenkes bei Hunden und Katzen. Ergebnisse einer Langzeitstudie an der Chirurgischen Veterinärklinik der Justus-Liebig-Universität Gießen. Tierärztliche Praxis 1997; 25(4): 273-378

Waldvogel HH. Analgetika, Antinozizeptiva, Adjuvanzien: Handbuch für die Schmerzpraxis. Heidelberg, Berlin: Springer Verlag; 2001

\section{Teil 2: Klinik und Diagnostik}

\section{Spezielle Erkrankungen}

Ursachen für die Entstehung einer sekundären felinen Osteoarthrose sind v.a. kongenitale und entwicklungsbedingte Erkrankungen und Gelenktraumata, aber auch infektiöse oder immunvermittelte Gelenkerkrankungen oder chronische Überbelastung.

Zu der Gruppe der kongenital- bzw. entwicklungsbedingten Erkrankungen gehört z.B. die Hüftgelenksdysplasie (HD) oder auch die Patellaluxation. Die Häufigkeit einer HD liegt bei der Katze je nach Studie zwischen 6 und $30 \%$ :

- Rassekatzen wie Perser, Maine Coon, Devon Rex, Himalaya oder Abessinier sind häufiger betroffen.

- Bei der Rasse Maine Coon finden sich studienabhängig sogar Prävalenzen bis zu über $70 \%$.

Nur ein geringer Anteil der betroffenen Tiere zeigt eine klinische Symptomatik.

Patellaluxationen können kongenital bzw. traumatisch durch eine Gelenkkapselruptur oder aber durch Gliedmaßenfehlstellungen nach Frakturen bedingt sein. Bei der kongenitalen Form ist die Patella meistens nach medial und oft auch bilateral luxiert.

Hingegen ist über das Auftreten einer Ellbogengelenksdysplasie bei der Katze wenig bekannt. In der Literatur wurde lediglich ein Fall beschrieben, bei dem das Vorliegen eines fragmentierten Processus coronoideus medialis ulnae vermutet wird.
Häufig führt ein Gelenktrauma zur Arthrosebildung. Zum einen kann es sich um eine Fraktur mit Gelenkbeteiligung handeln, die trotz operativer Versorgung zur Arthrose führen kann; zum anderen führen auch traumatische Gelenkinstabilitäten zu einer Osteoarthrose.

In diesem Zusammenhang sind v.a. das Hyperextensionssyndrom (Ruptur der palmaren Bänder des Karpalgelenks), Gelenkluxationen (z. B. Luxatio ossis femoris) und sonstige Rupturen von gelenkstabilisierenden Bändern zu nennen.

Arthritiden können ebenfalls Ursache einer Osteoarthrose sein. Man unterscheidet infektiöse und immunbedingte Arthritiden. Infektiös bedingte Arthritiden können durch Bakterien hervorgerufen werden, die via penetrierende Verletzungen, während eines chirurgischen Eingriffs, über eine angrenzende Osteomyelitis oder hämatogen in ein Gelenk eindringen können. Viren und Pilze sind ebenfalls imstande, eine Arthritis bei der Katze hervorzurufen. Hierzu zählen das Feline Leukose-Virus (FeLV), das Feline Synzytium-Forming Virus (FeSFV), Caliciviren, Histoplasma capsulatum und Cryptococcus neoformans.

Immunassoziierte Arthritiden kommen bei der Katze im Vergleich zum Hund selten vor. Man unterscheidet erosive und nichterosive Formen. Zu den nicht-erosiven Formen gehört der Systemische Lupus erythematodes(SLE), die idiopathische Polyarthritis und die medikamenteninduzierte Polyarthritis. Der SLE ist eine seltene, systemische
Erkrankung bei der Katze und ist durch das Vorhandensein von antinukleären Antikörpern gekennzeichnet.

Um eine idiopathische Polyarthritis (IP) handelt es sich, wenn keine der anderen Formen diagnostiziert werden kann. Die IP ist die am häufigsten vertretene immuninduzierte Arthritis beim Hund, während sie bei der Katze selten vorkommt. Sie lässt sich in 4 Formen unterteilen:

- Typ I: unkomplizierte Polyarthritis

- Typ II: reaktive IP, assoziiert mit Infektionen an irgendeiner Körperstelle

- Typ III: IP, assoziiert mit gastrointestinalen Störungen

- Typ IV: IP, assoziiert mit Neoplasien

Bei den erosiven immuninduzierten Polyarthritiden wird zwischen einem häufiger vorkommenden periostalen proliferativen Typ und einem selteneren rheumatoiden Typ unterschieden. Die periostale proliferative Polyarthritis ist eine destruktive Gelenkentzündung mit chronischem Verlauf, die überwiegend bei jungen männlichen Katzen unter 5 Jahren auftritt. Die Ursache ist unklar, man vermutet jedoch eine Verbindung mit dem Feline Synzytium-Forming Virus und dem Felinen Leukose-Virus.

Fraglich ist auch der Einfluss von Übergewicht auf die Arthroseentstehung bei der Katze. Dieser Zusammenhang ist für Hunde und auch den Menschen erwiesen, aber es gibt derzeit keine Veröffentlichungen, die dieses bei der Katze belegen. 
Lediglich der Zusammenhang zwischen Übergewicht und Lahmheit wurde untersucht. Dabei wurde festgestellt, dass das Risiko, wegen einer Lahmheit tierärztliche Hilfe in Anspruch zu nehmen, bei adipösen Katzen 4,9-mal höher ist als bei normalgewichtigen Tieren.

\section{Anamnese}

Im Gegensatz zum Hund stellt die Lahmheit bei der Katze nicht das häufigste Symptom im Rahmen der OA dar. Katzen haben die Fähigkeit, Anzeichen einer Erkrankung zu maskieren und keinerlei offensichtliche klinische Symptome zu zeigen.

\section{Verhaltensänderungen sind oft das auffälligste Symptom.}

Die Anamnese ist daher das wichtigste Instrument, um die Diagnose Osteoarthrose zu stellen. Sie sollte Fragen bezüglich klinischer Symptome wie auch Fragen bezüglich des Verhaltens der Tiere beinhalten.

Zu Beginn der Anamnese sollten Fragen nach dem Auftreten von Lahmheiten oder sonstigen Ganganomalien gestellt werden. Wichtig ist es auch, zu erfragen, ob Traumata bzw. Unfälle oder orthopädische Vorerkrankungen bestanden, da diese zur Arthroseentstehung führen können.

Manche Tiere mit OA zeigen aufgrund einer verminderten Bewegungsaktivität eine Gewichtszunahme, andere Tiere hingegen verlieren an Gewicht, da sie ihre Fütterplätze schmerzbedingt nicht mehr so häufig aufsuchen. Viele an OA erkrankte Tiere passen ihre Lebensweise der veränderten Situation an, indem sie weniger spielen, den Kratzbaum nicht mehr benutzen, frühere Lieblings- und Schlafplätze nicht mehr bzw. nicht mehr so häufig aufsuchen, nicht mehr jagen gehen oder auf Bäume klettern.

Auch das Kot- und Harnabsatzverhalten kann beeinträchtigt sein, sodass die Tiere die Katzentoilette nicht mehr aufsuchen bzw. nicht mehr in diese gelangen und Kot bzw. Harn neben der Toilette absetzen.

Weitere mögliche Anzeichen für Schmerzzustände bei der Katze sind:

- aggressives oder ängstliches

Verhalten

- erhöhter Schlafbedarf
- vermehrte Lautäußerungen

- veränderte soziale Verhaltensweisen

Es ist wichtig, explizit nach diesen

Verhaltensweisen zu fragen, da für

viele Tierbesitzer die mit der OA

einhergehenden Veränderungen als normale altersbedingte Veränderungen interpretiert werden.

Viele Tierbesitzer sind zudem der Ansicht, dass eine verminderte körperliche Aktivität für eine ältere Katze durchaus normal ist. Spezifische Schmerzanamnesebögen können daher für die Diagnosestellung sehr hilfreich sein, sind aber bisher noch nicht auf ihre Aussagekraft validiert worden ( Tab. 1).

\section{Klinische Untersuchung}

Die klinische Untersuchung bei Patienten mit Arthroseverdacht beinhaltet eine ausführliche allgemeine Untersuchung und eine spezielle Untersuchung des Bewegungsapparates.

Grundsätzlich ist anzuraten, Termine für Katzen in gering frequentierten Praxiszeiten einzuplanen, eine separate Katzensprechstunde oder ein Wartezimmer für Katzen einzurichten, da dies zu einer Reduktion des Stresslevels der Patienten führen kann. Viele Katzen sind bereits schon vor der Untersuchung z.B. durch den Transport oder die fremde Umgebung hochgradig gestresst.

Stress und Angst können zu einer Absenkung der Schmerzschwelle führen. Daher legen gestresste Katzen häufig ein aggressives und überempfindliches Verhalten an den Tag bzw. sind so angespannt, dass die Durchführung und Interpretation einer Untersuchung erschwert bzw. unmöglich ist.

Zu Beginn sollte die Analyse des Gangbildes erfolgen, da der Patient noch nicht zusätzlich durch die Untersuchung gestresst ist. Des Weiteren kann es sinnvoll sein, der Katze Zeit zur Akklimatisierung zu geben oder gar eine verdeckte Beobachtung durchzuführen, z. B. indem das Tier durch ein Fenster beobachtet wird.

Weitere Hinweise auf eine mögliche Osteoarthrose:
Tab. 1 Spezielle anamnestische Fragen an den Katzenbesitzer zur Unterstützung der Diagnosestellung von Erkrankungen des Bewegungsapparates (modifiziert nach Lindley).

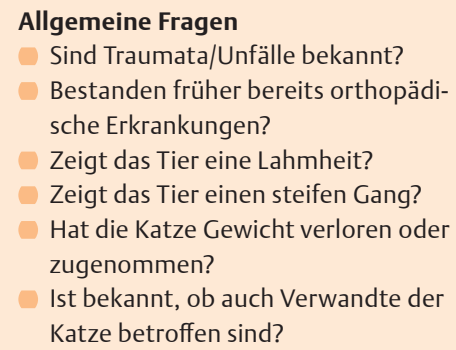

Sind Ihnen Veränderungen bei folgenden Aktivitäten aufgefallen?

- Treppe hinauf- und/oder hinuntergehen

- Spielen

- Benutzung des Kratzbaumes

Springen oder Klettern auf Fensterbank, Schoß, Tisch etc.

Klettern auf Bäume, Zäune

Sind Ihnen Veränderungen bei folgenden Verhaltensweisen aufgefallen?

- Kot-/Urinabsatz

zurückgezogene Lebensweise

Aggressivität Menschen/Artgenossen gegenüber

- Fellpflege (reduziert oder vermehrtes Lecken)

- Schlafgewohnheiten

Schmuseverhalten

Lautäußerungen

Katzen mit dieser Erkrankung vernachlässigen häufig ihre Fell- und Körperpflege, sodass sie mit einem stumpfen und matten Fell vorgestellt werden.

- Teilweise können auch haarlose Körperstellen im Bereich der Gelenke festgestellt werden, was Ausdruck von vermehrtem Lecken als Folge von Hyperästhesien sein kann.

- Lange Krallen lassen auf eine reduzierte körperliche Aktivität bzw. ein reduziertes Kratzverhalten schließen.

Arthrotische Gelenke:

- Sie sind häufig verdickt; dies kann zum einen Ausdruck einer Gelenkfüllung sein oder zum andern auch durch eine Kapselverdickung hervorgerufen werden. 
- Beim passiven Beugen und Strecken der erkrankten Gelenke können oft eine Pseudokrepitation, ein eingeschränkter Bewegungsradius (Range of Motion) und eine Schmerzhaftigkeit festgestellt werden.

Gliedmaßen mit arthrotischen Gelenken:

- Die Muskulatur an der erkrankten Gliedmaße kann aufgrund der Entlastung atrophiert bzw. die Muskulatur auf der gesunden Seite durch die Überbeanspruchung hypertrophiert sein.

Fazit: Die Interpretation der orthopädischen Untersuchung bei Katzen ist sehr schwierig

- Klinische Befunde sind oft weniger deutlich als beim Hund, insbesondere Pseudokrepitation und ein eingeschränkter Bewegungsradius sind nur selten im Rahmen der Untersuchung festzustellen.

- Es besteht ein deutlicher Unterschied zwischen Befunden der orthopädi- schen und der radiologischen

Untersuchung:

- Im Rahmen einer Veröffentlichung von Clarke und Bennett zeigten 34\% der in orthopädischer Untersuchung als schmerzhaft befundeten Gelenke keine radiologischen Hinweise auf das Vorliegen einer OA.

- Bei einer weiteren Studie wurde ebenfalls nur eine mittelgradige Übereinstimmung zwischen der Schmerzhaftigkeit bei Manipulation und der radiologischen Diagnose Arthrose festgestellt. Bei lediglich 33\% der Gelenke, bei denen radiologisch eine Arthrose diagnostiziert wurde, konnte bei der orthopädischen Untersuchung eine Schmerzhaftigkeit festgestellt werden.

\section{Weiterführende Diagnostik}

Ergeben sich durch Anamnese und klinische Untersuchung Hinweise auf das Vorliegen einer OA müssen zur Diagnosesiche- rung Röntgenaufnahmen der klinisch verdächtigen Gelenke in jeweils 2 Ebenen durchgeführt werden.

Sollte im Rahmen der klinischen Untersuchung kein schmerzhaftes Gelenk eindeutig lokalisiert werden können, kann man ggf. Aufnahmen der Hüft-, Ellbogen- und Kniegelenke anfertigen, da diese Gelenke am häufigsten betroffen sind.

Das Röntgen ist ein zuverlässiges und kostengünstiges Diagnostikum, das vielen Praxen zur Verfügung steht. Um die Röntgenaufnahmen richtig interpretieren zu können, ist eine korrekte Lagerung des Patienten unabdingbar.

Viele Katzen, insbesondere schmerzhafte Tiere, tolerieren dies nicht ohne Weiteres, sodass man ggf. eine Sedierung durchführen sollte. Folgende Kombinationen stehen zur Verfügung:

- Butorphanol $(0,4 \mathrm{mg} / \mathrm{kg}) /$ Medetomi$\operatorname{din}(0,05 \mathrm{mg} / \mathrm{kg})$ s.c. oder i.m 

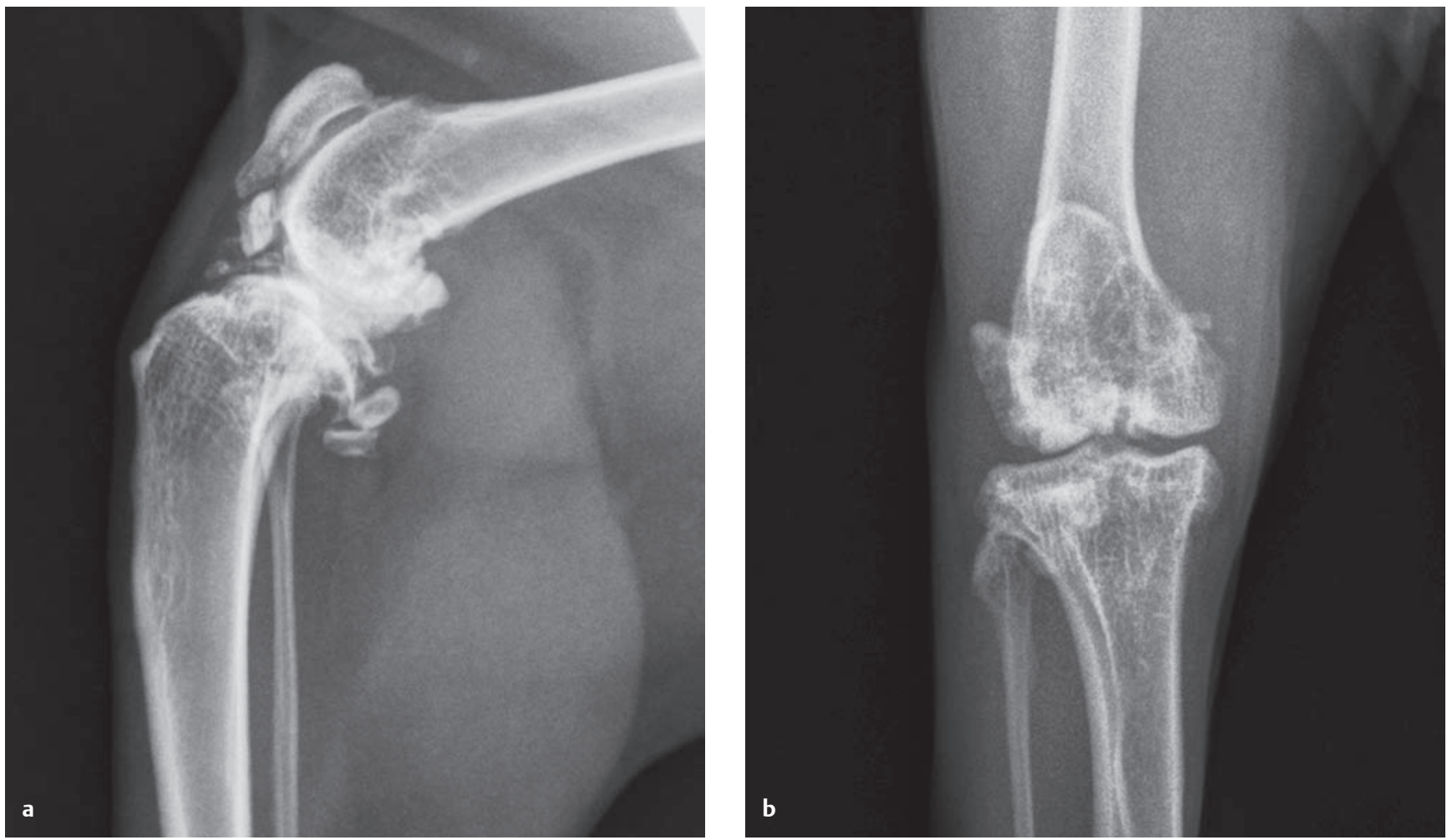

Abb. 3 Röntgenaufnahme des rechten Kniegelenks einer Katze mit Gonarthrose im medio-lateralen (a) und kranio-kaudalen (b) Strahlengang. Man erkennt die hochgradigen osteophytären Zubildungen in allen Gelenksanteilen, intraartikuläre Mineralisationen, subchondrale Sklerosierung, KnochenRemodeling und einen vermehrten intraartikulären Weichteilschatten.

- Diazepam (0,5 mg/kg)/Propofol (2-4 $\mathrm{mg} / \mathrm{kg})$ i.v.

- Medetomidin $(0,08 \mathrm{mg} / \mathrm{kg}) /$ Ketamin $(1 \mathrm{mg} / \mathrm{kg}$ ) s.c. oder i.m.

Die Kombinationen von Medetomidin mit Butorphanol oder der niedrigen Dosis von Ketamin bieten den Vorteil, dass eine sofortige Antagonisierung mittels Atipamezol möglich ist.

Typische röntgenologische Veränderungen einer Osteoarthrose ( Abb. 3) sind:

- Osteophyten- und Enthesiophytenbildung

- subchondrale Sklerosierung oder Zysten

- Knochen-Remodeling

- Gelenkspaltverschmälerung

- Gelenkfüllung

- periartikuläre Weichteilschwellung

- intraartikuläre Mineralisationen („loose bodies“)

Bei der Interpretation ist zu beachten, dass der Grad der radiologischen Veränderun- gen nicht mit der klinischen Relevanz korreliert. Das Röntgen ist zwar gut geeignet, um knöcherne Veränderungen zu detektieren, Veränderungen des Gelenkknorpels oder des Stratum synoviale können hingegen nicht dargestellt werden.

Es ist möglich, dass hochgradige röntgenologische Veränderungen keine klinische Bedeutung haben, aber auch dass röntgenologisch unauffällige Gelenke hochgradig schmerzhaft sind. Vor diesem Hintergrund ist die Röntgenuntersuchung nur in Verbindung mit einer ausführlichen Anamnese und einer klinischen Untersuchung als Hilfsmittel auf dem Weg zur Diagnose der felinen Osteoarthrose zu sehen (Triangulation).

Des Weiteren können durch die Röntgenuntersuchung Ursachen für das Auftreten einer Arthrose, wie z.B. eine Hüftgelenksdysplasie, erkannt werden.

Um die Ursache einer sekundären Arthrose feststellen zu können, kann ebenfalls die Durchführung einer Computertomografie oder auch einer Magnetresonanztomografie sinnvoll sein.
Wenn sich sowohl bei der klinischen als auch bei der radiologischen Untersuchung keine Hinweise auf die Lahmheitsursache ergeben, besteht die Möglichkeit der Szintigrafie. Die Szintigrafie ist ein bildgebendes Verfahren der nuklearmedizinischen Diagnostik. Dabei werden radioaktiv markierte Stoffe appliziert, die sich im erkrankten Zielorgan anreichern und anschließend mit einer Gammakamera, von der die abgegebene Strahlung aufgefangen wird, sichtbar gemacht werden können. Diese Methode eignet sich zur Lokalisationsdiagnostik z.B. von Entzündungsherden des Skelettsystems.

Eine Gelenkpunktion mit anschließender bakteriologischer und zytologischer Untersuchung der Synovia ist bei Katzen mit OA nur selten angezeigt. Mögliche Indikationen sind die Beteiligung mehrerer Gelenke, das Vorhandensein von Gelenkergüssen oder wenn bei jüngeren Tieren eine traumatische oder entwicklungsbedingte Ursache nicht erkennbar ist. 


\section{Teil 3: Therapie}

Da die bereits am Gelenkknorpel entstandenen Schäden irreparabel sind, ist eine Heilung im eigentlichen Sinne nicht möglich. Bei der Behandlung der Arthrose geht es v.a. darum, die Lebensqualität des Patienten zu verbessern und auf einem hohen Niveau zu halten. Hierzu werden folgende Therapieziele verfolgt:

- Kontrolle der Schmerz- und Entzündungsreaktionen

- Erhaltung bzw. Verbesserung der Bewegungsfähigkeit der erkrankten Gelenke

- Verlangsamung bzw. Stoppen des Fortschreitens der Erkrankung

Es ist wichtig, den Tierbesitzer schon am Anfang der Therapie dahingehend aufzuklären, dass sehr oft eine lebenslange Therapie notwendig ist und dass es im Verlauf der Erkrankung immer wieder zu akuten Schüben mit Schmerzen und daher einer Phase der intensiveren Therapie kommen kann.

Grundsätzlich wird bei der Therapie der Osteoarthrose zwischen operativen und konservativen Therapieverfahren unterschieden.

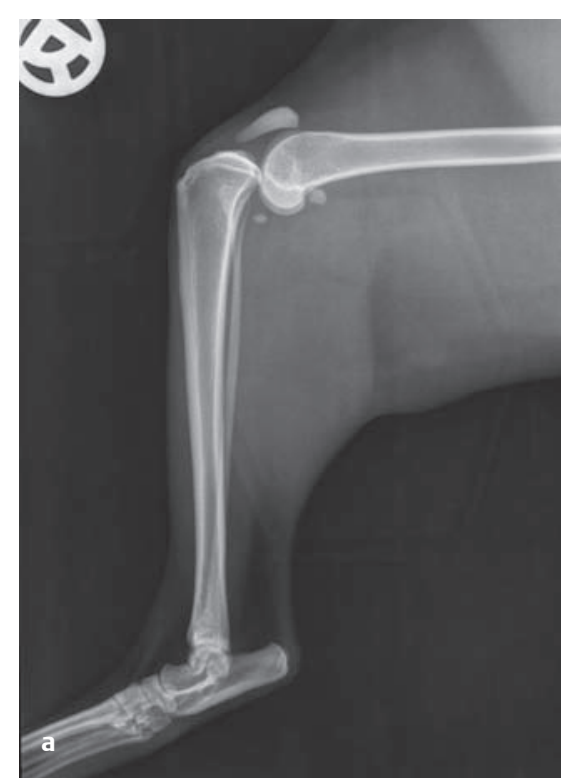

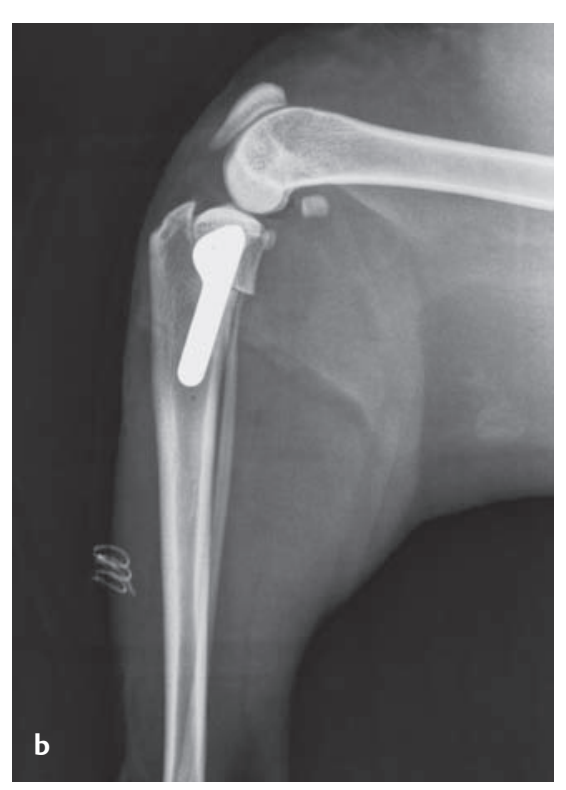

\section{Chirurgische Therapie}

Schon nach der Diagnosestellung der OA muss überlegt werden, ob chirurgische Maßnahmen notwendig und therapeutisch sinnvoll sind. Typische Indikationen für die chirurgische Therapie sind v.a. Gelenkinstabilitäten (z.B. Ruptur eines Kollateralbandes) und Coxarthrosen.

Die am häufigsten eingesetzten chirurgischen Verfahren kann man grundsätzlich in solche einteilen, die

- die zugrunde liegenden Ursachen beseitigen,

- das Gelenk versteifen oder

- das betroffene Gelenk entfernen.

$\mathrm{Zu}$ den Verfahren, die die Ursachen der Arthroseentstehung beseitigen, gehören u.a. stabilisierende Techniken (z.B. Bandersatz nach Ruptur eines Seitenbandes), aber auch Techniken, die die Biomechanik eines Gelenks verändern.

Zu letzteren Verfahren gehört z. B. die sog. Tibial Plateau Leveling Osteotomie (TPLO). Die TPLO ist ein Verfahren, das beim Hund schon seit geraumer Zeit zur
Versorgung einer Ruptur des kranialen Kreuzbandes erfolgreich eingesetzt wird und auch bei der Katze eingesetzt werden kann ( Abb. 4).

Bei der Katze liegt jedoch nur selten eine solitäre Ruptur des kranialen Kreuzbandes vor, häufiger handelt es sich um ein polytraumatisiertes Kniegelenk mit zusätzlichen Seitenbandrupturen, Meniskusschäden und Weichteiltraumata.

Ziel der stabilisierenden Verfahren ist es, v.a. das Fortschreiten der Arthrose zu stoppen bzw. zu verlangsamen.

Als Ultima Ratio besteht bei einigen Gelenken auch die Möglichkeit der Arthrodese oder aber der Resektionsarthroplastik. Am häufigsten wird die Femurkopfhalsresektion (FKHR) durchgeführt.

Indikationen zur FKHR sind neben einer hochgradigen Coxarthrose u.a. auch Azetabulumfrakturen und Femurkopfluxationen.

Der Oberschenkelkopf und -hals wird mittels Osteotomie von medial des

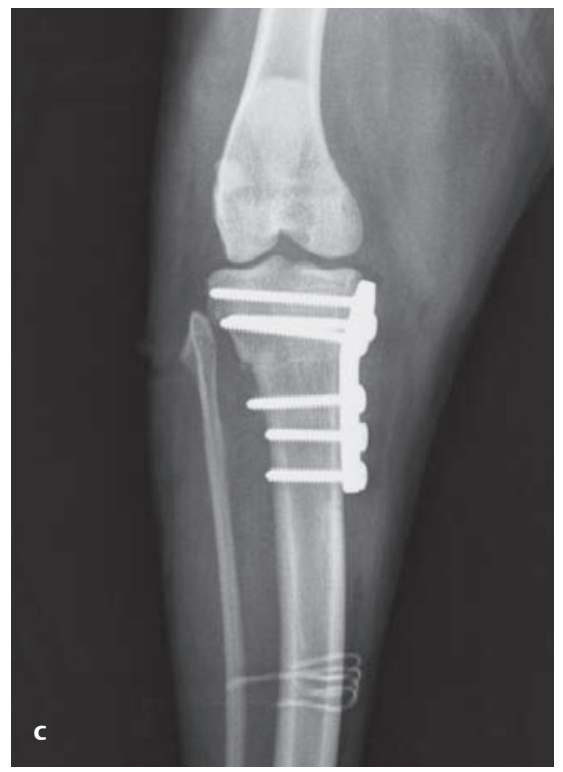

Abb. 4 a Röntgenaufnahme des rechten Kniegelenks einer Katze mit einer Ruptur des kranialen Kreutzbandes und des medialen Kollateralbandes nach unbekanntem Trauma im medio-lateralen Strahlengang. Man erkennt eine deutliche Luxationsstellung der Tibia nach kranial und einen vermehrten intraartikulären Weichteilschatten. b Röntgenaufnahme des in Abb. 4a beschriebenen Kniegelenks im medio-lateralen Strahlengang nach Versorgung der Ruptur des kranialen Kreuzbandes mittels TPLO. Die Enden des rupturierten medialen Kollateralbandes wurden adaptiert. c Röntgenaufnahme des in Abb. 4a beschriebenen Kniegelenks im kranio-kaudalen Strahlengang nach Versorgung der Ruptur des kranialen Kreuzbandes mittels TPLO. 
Trochanter major bis zum Trochanter minor entfernt. Im späteren Verlauf kommt es zur Ausbildung einer Syndesmose ( Abb. 5).

Unter Arthrodese wird eine permanente Versteifung eines Gelenks verstanden. Diese Technik wird v.a. nach Luxationen im Karpal- oder Tarsalgelenk durchgeführt, kann dort aber auch bei Tieren mit Osteoarthrose eingesetzt werden. Zur Fixation wird meistens eine stabile, gelenkübergreifende Plattenosteosynthese vorgenommen. Die Verwendung eines Fixateur externe oder von Schrauben und Nägeln ist situationsabhängig ebenfalls möglich.

\section{Konservative Therapie}

\section{Medikamentöse Schmerztherapie}

Die medikamentöse Schmerztherapie ist eine effektive Behandlungsoption für Katzen mit OA, bei denen keine Indikation zur Operation besteht. Ansonsten ist sie eine wichtige ergänzende Therapie.

\section{NSAIDs}

Im Vordergrund der medikamentösen Schmerztherapie stehen die Cyklooxigenasehemmer. Sie gehören zu der Gruppe der nichtsteroidalen Antiphlogistika (NSAIDs). NSAIDs wirken peripher antiinflammatorisch, analgetisch, aber auch antipyretisch und antithrombotisch und sind potente Inhibitoren der Prostaglandin- und vereinzelt auch der Leukotriensynthese.

Ein potenzielles Problem bei der Behandlung einer OA bei der Katze ist die Tatsache, dass Katzen sehr anfällig für die toxischen Wirkungen von NSAIDs sind. Ursächlich ist die geringe Glukuronidierungskapazität der Katzenleber.

Für die Behandlung chronischer Erkrankungen des Bewegungsapparates kann Meloxicam (z.B. Metacam ${ }^{\circledR}$, präferenzieller COX-2-Hemmer) oder auch Robenacoxib (Onsior ${ }^{\circledR}$, selektiver COX-2-Hemmer) bei der Katze erfolgreich eingesetzt werden. $\mathrm{Zu}$ beachten ist allerdings, dass man die Wirkung eines NSAIDs erst nach einer Applikationsdauer von mindestens 4 Wochen beurteilen kann, aber Onsior ${ }^{\circledR}$ nur eine Zulassung für einen Anwendungszeitraum von 6 Tagen besitzt.

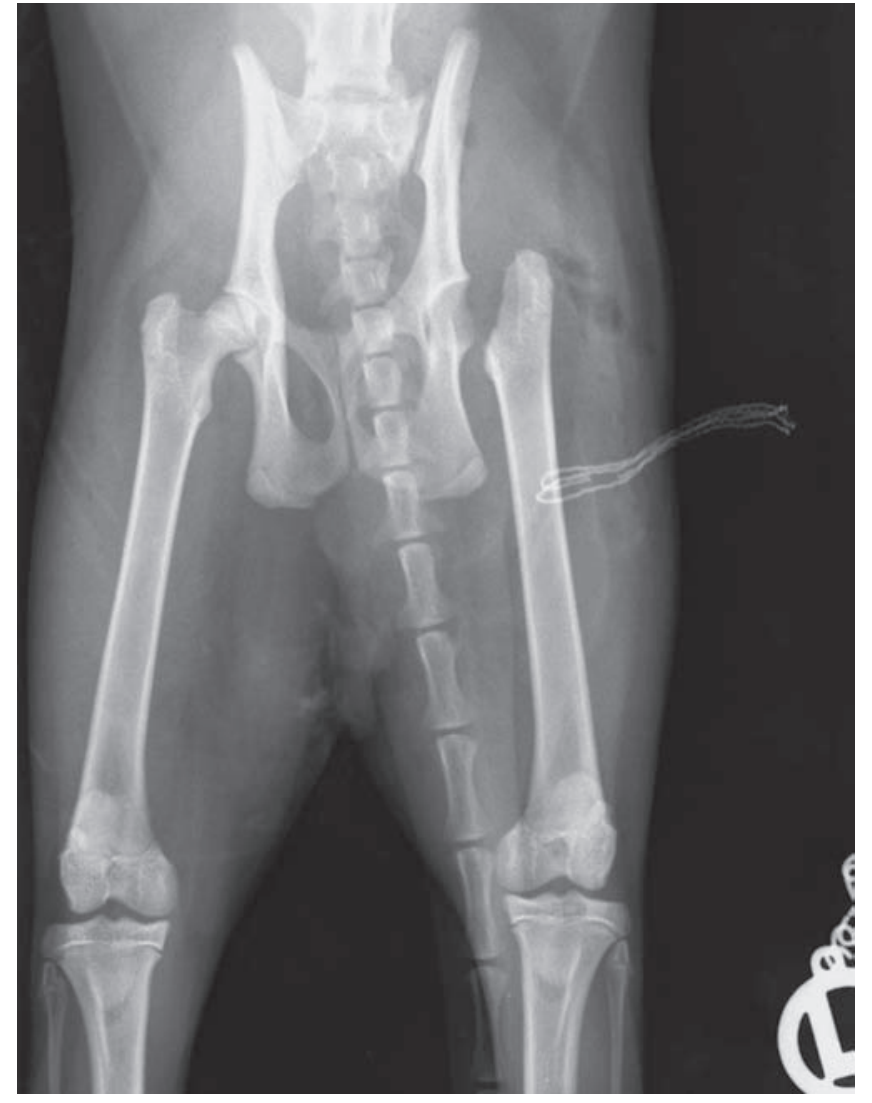

Abb. 5 Röntgenbild des Beckens einer Katze im ventro-dorsalen Strahlengang nach Femurkopfhalsresektion.

Wenn nötig kann versucht werden, die zu verabreichende Dosis zu reduzieren:

z.B. Metacam auf $0,025 \mathrm{mg} / \mathrm{kg}$ alle

24-72 h (anstatt $0,05 \mathrm{mg} / \mathrm{kg}$ alle $24 \mathrm{~h}$ )

Dabei ist unbedingt darauf zu achten, dass die Tierbesitzer aufgeklärt werden, dass bei den geringsten Anzeichen von Schmerzen oder Nebenwirkungen eine Wiedervorstellung beim Tierarzt erfolgen muss. Beim Auftreten von Nebenwirkungen wie z.B. Durchfall, Erbrechen, Polyurie oder Polydipsie muss die Therapie unterbrochen werden.

Vor Therapiebeginn und im Verlauf einer Langzeittherapie sollte eine labordiagnostische Untersuchung der Nieren- und Leberwerte erfolgen (Blut- und Urinuntersuchung). Therapiebegleitend ist auf eine ausreichende Flüssigkeitszufuhr des Patienten zu achten. Diese kann z. B. über das Aufstellen mehrerer Wassernäpfe oder das Verfüttern von Feuchtfutter gesteigert werden.

\section{Glukokortikoide}

Die Verwendung von Glukokortikoiden zur Behandlung der felinen Osteoarthrose ist umstritten. Glukokortikoide wirken anti- inflammatorisch, analgetisch und antipyretisch.

Trotz ihrer Wirkqualität gelten Glukokortikoide im Rahmen einer Langzeittherapie als risikoreicher im Vergleich zu NSAIDs, da ihre Anwendung zu systemischen Nebenwirkungen führen kann.

Sie sollten systemisch nur zum Einsatz kommen, wenn andere Therapieversuche erfolglos bleiben und dann sollte möglichst schnell die therapeutische Mindestdosis ermittelt werden, um das Ausmaß der Nebenwirkungen zu minimieren.

Eine gleichzeitige Anwendung mit NSAIDs ist kontraindiziert, da es sonst zu schweren gastrointestinalen Nebenwirkungen kommen kann.

Des Weiteren besteht die Möglichkeit, ein langsam zu resorbierendes Glukokortikoid intraartikulär zu injizieren. Damit es nicht zu einer Schädigung des Gelenkknorpels kommt, sollte die Injektion nicht so oft wiederholt werden. In der Humanmedizin gilt, dass die Injektionen in wöchentlichen bis 4-wöchentlichen Abständen durchgeführt werden. 
Die Gesamtzahl von 4 Injektionen pro Jahr sollte nicht überschritten werden.

\section{Slow Acting Drugs in Osteoarthritis (SADOA) und Ergänzungsfuttermittel} Zu der Gruppe der SADOA gehören Substanzen wie Glukosaminsulfat, Chondroitinsulfat und Hyaluronsäure, die seit vielen Jahren zur symptomatischen Behandlung der OA beim Menschen und Hund eingesetzt werden.

Sie sollen den Krankheitsverlauf verlangsamen und gleichzeitig auch zur Schmerzlinderung beitragen. Hintergrund für die Anwendung dieser Wirkstoffe ist die Tatsache, dass sie Vorläufersubstanzen für die Synthese und Reparatur des Gelenkknorpels sind und zu einer Verlangsamung des Knorpelabbaus führen sollen.

Es sind zurzeit einige Nahrungsergänzungsprodukte, sog. Nutraceuticals, auf dem Markt, die bei Katzen mit Problemen des Bewegungsapparates eingesetzt werden können (z.B. Cosequin ${ }^{\circledR}$, Caniviton ${ }^{\circledR}$, Canosan ${ }^{\circledR}$ Katze).

Ebenfalls stehen Spezialnahrungsmittel (functional foods) für Katzen mit Mobilitätsproblemen zur Verfügung, die u.a. häufig Glukosamine, Glukosaminoglykane (GAGs), Hyaluronsäure und Chondroitinsulfat enthalten.

Diese Stoffe sollen die Knorpelsynthese fördern. Viele Produkte enthalten auch bestimmte Fettsäuren (z. B. Eicosatetraensäure, Docosahexaensäure), Antioxidanzien wie die Vitamine C und E (z.B. Royal Canin Mobility $^{\circledR}$ ).

Außerdem kommen Extrakte der Grünlippenmuschel (enthält u.a. GAGs, Omega3-Fettsäuren), Zink, Mangan, Selen, Avocado-, Soja-, Weihrauch-, Weidenrinde-, Pfeffer-, Artischocken-, Wintergrün-, Katzenkrallen-, Teufelskrallen-, Ingwer-, Maulbeer- und Vanilleextrakte zum Einsatz. In einer veterinärmedizinischen Literaturübersicht, bei der auch die wissenschaftliche Qualität der Studien betrachtet wurde, konnte für diese Präparate keine eindeutige Wirkungsüberlegenheit beim Hund beobachtet werden. Dies bedeutet nicht, dass diese Substanzen keinen positiven Effekt auf das Arthrosegeschehen haben können, sondern nur, dass der wissenschaftliche Nachweis fehlt. Derzeit liegen keine objektiven Daten bezüglich der Wirksamkeit bei der Katze vor.

\section{Physiotherapie}

Die Physiotherapie ist eine Behandlungsform unter Anwendung physikalischer Mittel. Darunter fallen z. B.:

- Bewegung (Mobilisierung, Stretching, Bewegungsübungen, Massagen)

- thermische Elemente (Kälte, Wärme)

- elektrischer Strom (z. B. Transkutane elektrische Nervenstimulation TENS)

- Schallwellen (therapeutischer Ultraschall)

- Licht (Laser)

- Magnetfelder

- extrakorporale Stoßwellen
Häufig im Rahmen der OA angewendete Verfahren sind die passive Gelenkmobilisierung, Stretching, aktive therapeutische Übungen (z.B. Treppensteigen, Hindernisparcour, ,Sitz-Aufsteh“-Übungen),Schwimmtherapie und Massage.

Regelmäßige Physiotherapie kann helfen, den Bewegungsumfang eines Gelenks zu vergrößern. Grundvoraussetzung ist jedoch, dass die erkrankten Tiere die Durchführung dieser Maßnahmen zulassen. Und hier gibt es gerade bei der Katze häufiger Probleme.

\section{Gewichtsmanagement}

Ein Zusammenhang zwischen Übergewicht und Arthroseentstehung ist bei Katzen, im Gegensatz zum Hund, bisher nicht nach- 


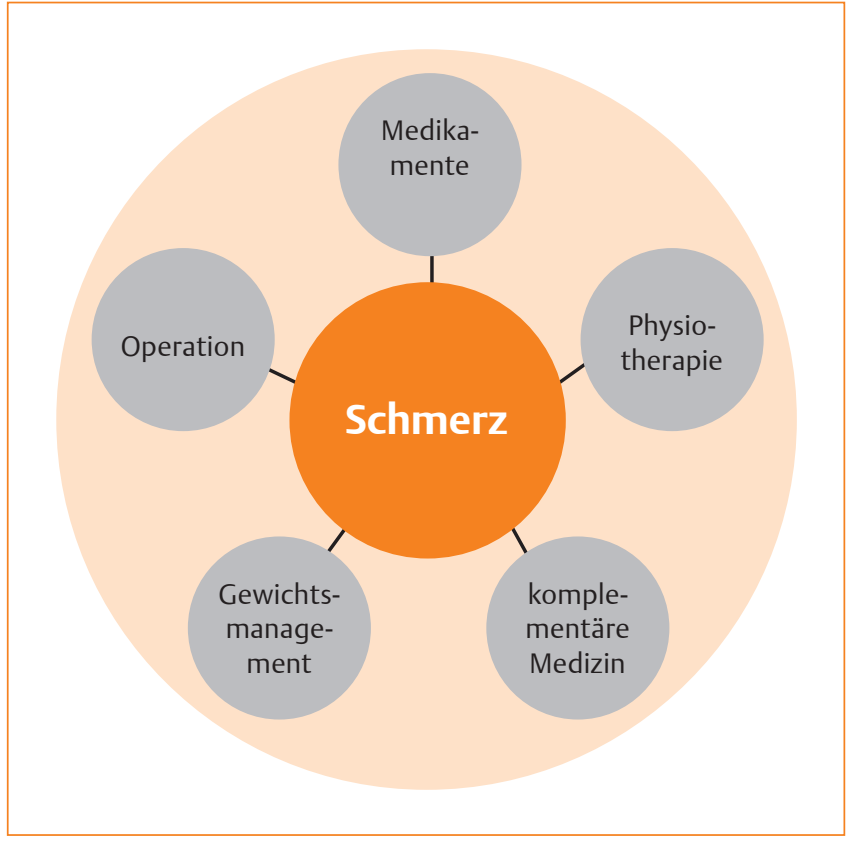

Abb. 6 Schema: Multimodale Schmerztherapie.

gewiesen. Dennoch sollte ein Adipositasmanagement bei entsprechender Indikation durchgeführt werden. Hierzu zählt u.a. die regelmäßige Gewichtskontrolle, Reduktion der Energiezufuhr und vermehrte Bewegung (z.B. Animation zum Spiel).

Bei manchen Tieren mit OA, v.a. älteren Tieren, ist nicht das Übergewicht problematisch, sondern eher die vermehrte Gewichtsabnahme. In solchen Fällen ist es sinnvoll, Futter und Trinkwasser an mehreren gut erreichbaren Lokalisationen anzubieten, da dies zu einer erhöhten Aufnahme führen kann. Auch in solchen Fällen ist eine regelmäßige Gewichtskontrolle anzuraten.

\section{Anpassung der Haltungsbedingungen}

Die Haltungsbedingungen sollten individuell an das erkrankte Tier angepasst werden. Die Ruheplätze des Tieres sind zum
Schutz der Gelenke weich zu polstern. Wenn die Katze gewohnte Aufenthaltsplätze (z.B. Fensterbank, erhöhter Schlafplatz) nicht mehr aufsuchen kann, sollte es dem Tier erleichtert werden, diese $\mathrm{zu}$ erreichen. Dies kann durch Rampen oder aber auch durch die Einrichtung von Zwischenstationen erzielt werden.

Wird die Katzentoilette aufgrund der OA nicht oder nur noch vereinzelt aufgesucht, kann die Bereitstellung von mehreren Toiletten mit niedrigen Seitenwänden (erleichterter Einstieg) an verschiedenen Bereichen in der Wohnung eine Lösung des Problems bedeuten.

\section{Klinische Schlussfolgerung}

Da die Hauskatzen heutzutage immer älter werden, nimmt die feline OA eine zunehmend größere Stellung in der Katzenpo- pulation ein. Das Erkennen dieser Erkrankung stellt immer noch ein großes Problem dar, da erkrankte Tiere keine klassischen Lahmheitssymptome zeigen, sondern häufig nur Verhaltensänderungen an den Tag legen. Viele Tierbesitzer deuten diese Verhaltensänderungen häufig als normale altersbedingte Veränderungen. Eine wichtige Aufgabe der Tierärzte ist es, die Besitzer über die $\mathrm{OA}$ zu informieren und für ein Erkennen der Symptome zu sensibilisieren.

Die Diagnosestellung erfolgt i.d.R. durch eine gründliche Anamnese in Kombination mit einer orthopädischen und einer röntgenologischen Untersuchung, wobei die Anamnese das wertvollste diagnostische Werkzeug für die Identifizierung von an Arthrose erkrankten Katzen ist.

Derzeit stehen eine Vielzahl verschiedener Managementstrategien und medikamentöser Behandlungsoptionen zu Verfügung. Daher ist es wichtig, die Therapie und das Management individuell auf den jeweiligen Patienten anzupassen. Die Arthrosebehandlung sollte multimodal sein, d.h., es sollten unterschiedliche Therapieverfahren, wie z.B. die operative Versorgung, medikamentöse Schmerztherapie, Gewichtsmanagement oder Physiotherapie, in Kombination zum Einsatz kommen ( Abb. 6).

Online zu finden unter

http://dx.doi.org/10.1055/s-0031-1275559

\section{Alexander Acker}

PD Dr. Sabine Tacke

Klinik für Kleintiere (Chirurgie)

Justus-Liebig-Universität

Frankfurter Str. 108 · 35392 Gießen

E-Mail: Sabine.P.Tacke@vetmed.uni-giessen.de 


\section{Fragebogen \\ Fragen zu Teil 1-3}

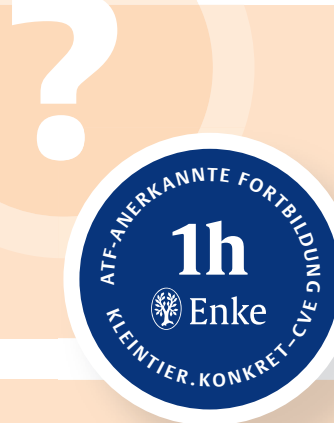

\section{Frage 1}

\section{Wann ist die Durchführung einer}

\section{Szintigrafie indiziert?}

a. Zur Einteilung der Arthrosegrade

b. Zur Einteilung des Typs der immuninduzierten Arthritis

c. Zur näheren Differenzierung von traumatischen Gelenkinstabilitäten

d. Zur Lokalisationsdiagnostik von Entzündungsherden des Skelettsystems

e. Zur Einordnung des Grads einer Patellaluxation

\section{Frage 2}

Warum ist die Katze anfälliger für die toxische Wirkung von NSAIDs als der

Hund?

a. Bei der Katze liegt sehr häufig der MDR-1Defekt vor.

b. Die Leber der Katze besitzt eine geringere Glukuronidierungskapazität.

c. Die Katze kann keine Xenobiotika ausscheiden.

d. Die Biotransformation durch Konjugation ist bei der Katze in der Niere nur eingeschränkt möglich.

e. Die Gabe von NSAIDs ist bei der Katze grundsätzlich mit der Bildung von Methämoglobin verbunden.

\section{Frage 3}

\section{Welche Aussage bezüglich der felinen}

\section{Osteoarthrose trifft zu?}

a. Die Mehrzahl der Osteoarthrosefälle bei der Katze sind primären Ursprungs.

b. Katzen mit Osteoarthrose zeigen als klinische Symptomatik meistens eine Lahmheit.

c. Die Ursache der Osteoarthrose bei der Katze besteht immer in einer traumatischen Gelenkinstabilität.

d. Die Osteoarthrose bei der Katze ist meist die Folge von infektiösen Arthritiden.

e. Bei der Katze ist der Einfluss von Adipositas auf die Arthroseentstehung wissenschaftlich belegt.

\section{Frage 4}

Was ist eine typische radiologisch sichtbare Veränderung beim Vorliegen einer Osteoarthrose?
a. Subluxation
b. Fissurlinien
c. Enthesiophytenbildung

d. Kallusbildung

e. Luxation

\section{Frage 5}

Welche Aussage über den Knorpelmetabolismus trifft zu?

a. Interleukin 4 und Interleukin 6 wirken anabol auf den Knorpel.

b. Durch eine Synovialitis wird der Aufbau des Gelenkknorpels angeregt.

c. Matrix-Metalloproteasen kommen nur im Rahmen der Osteoarthrose vor.

d. IGF, TGF $\beta$ sind katabole Faktoren.

e. Matrix-Metalloproteasen spalten u.a. Proteoglykane und Kollagen.

\section{Frage 6}

Was muss bei der klinischen Untersuchung von Katzen mit Erkrankungen des Bewegungsapparates beachtet werden?

a. Transportstress und die neue Umgebung erleichtern die Interpretation der Untersuchung deutlich.

b. Die Befunde der orthopädischen Untersuchung sind bei Katzen immer eindeutig, und weitere Diagnostik ist nicht nötig.

c. Bei der speziellen orthopädischen Untersuchung muss die Katze immer in Narkose gelegt werden.

d. Die Befunde der orthopädischen Untersuchung sind bei der Katze meist dezenter als beim Hund.

e. Bei der speziellen orthopädischen Untersuchung kann zur Stressminimierung grundsätzlich auf eine Gangbildanalyse verzichtet werden.

\section{Frage 7}

Was muss der Tierbesitzer bezüglich der

Arthrosetherapie bei Katzen wissen?

a. Die Behandlung ist unkompliziert, und eine Heilung wird nach maximal 4 Wochen erreicht.

b. Falls an Arthrose erkrankte Katzen lediglich Verhaltensänderungen zeigen, reicht meist die einmalige Gabe eines NSAIDs in Kombination mit einer Verhaltenstherapie aus.

c. Die bereits am Gelenkknorpel entstandenen Schäden sind irreparabel, und eine Heilung im eigentlichen Sinne ist nicht möglich.

d. Bei der Gonarthrose ist eine Arthrodese die Therapiemethode der Wahl.

e. Die operative Arthrosetherapie ist immer indiziert.

\section{Frage 8}

Welche Form der idiopathischen Polyarthritis ist mit welchem Krankheitsbild assoziiert?

a. Typ II ist mit Neoplasien assoziiert.

b. Beim Typ I handelt es sich um eine umkomplizierte Polyarthritis.

c. Beim Typ IV handelt es sich um eine unkomplizierte Polyarthritis.

d. Es werden keine Typen unterschieden.

e. Typ III ist mit einer Infektion an irgendeiner Körperstelle assoziiert.

\section{Frage 9}

\section{Was ist bei der Diagnostik von Katzen} mit dem Verdacht auf Osteoarthrose zu beachten?

a. Explizites Erfragen von Verhaltensänderungen gibt wichtige Hinweise auf das Vorliegen einer Osteoarthrose.

b. Die Anamnese ist unnötig, wenn eine Ganzkörper-Röntgenaufnahme angefertigt wird.

c. Unter Triangulation versteht man die Verknüpfung der Szintigrafie mit der klinischen Untersuchung und der Gelenkpunktion zur Diagnosestellung der Osteoarthrose.

d. Das Schultergelenk ist das am häufigsten betroffene Gelenk.

e. Knorpeldegenerationen können auf Röntgenaufnahmen sicher erkannt werden.

\section{Frage 10}

Welcher selektive COX-2-Hemmer ist für die Anwendung bei Katzen zugelassen?
a. Robenacoxib
b. Meloxicam
c. Ketoprofen
d. Tolfenaminsäure
e. Piroxicam 


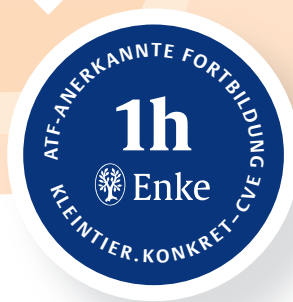

A Lernerfolgskontrolle

\section{Bitte kreuzen Sie die richtigen Antworten an! Es ist jeweils nur 1 Antwort pro Frage richtig!}

\section{B Teilnehmer}

Titel | Name | Vorname

Straße | Hausnummer

PLZ | Ort

\section{E Erklärung}

Ich versichere, dass ich die Beantwortung der Fragen selbst und ohne Hilfe durchgeführt habe.

Stempel | Unterschrift

kleintier konkret-CVE-Wertmarken für NichtAbonnenten können beim Verlag zu folgenden Bedingungen erworben werden: 6erPackWertmarken, Preis 49,95€ inkl. MWSt., Artikel-Nr. 903000.

Bitte richten Sie die Bestellungen an: MVS Medizinverlage Stuttgart,

KundenServiceCenter Buch, Postfach 3011 20, 70451 Stuttgart.

${ }^{*}$ Nicht-Abonnenten bitte hier kleintier konkret-CVE-Wertmarke aufkleben, Abonnenten bitte Abonnentennummer eintragen 